\title{
SPATIAL DISTRIBUTION OF STAR FORMATION ACTIVITY ON NGC 253 BY FIR AND RADIO EMISSION LINES
}

\author{
H. TAKahashi ${ }^{1}$, H. MatsuO ${ }^{2}$, And K. NAKAnishi ${ }^{3}$ \\ ${ }^{1}$ Institute of Astronomy, The University of Tokyo, 2-21-1 Osawa, Mitaka, Tokyo 181-0015, Japan \\ ${ }^{2}$ Advanced Technology Center, National Astronomical Observatory of Japan \\ ${ }^{3}$ ALMA Project Office, National Astronomical Observatory of Japan, Chile \\ E-mail: nori@ioa.s.u-tokyo.ac.jp \\ (Received July 01, 2012; Accepted August 16, 2012)
}

\begin{abstract}
The aim of this research is to reveal the spatial distribution of the star formation activity of nearby galaxies by comparing $\mathrm{CO}$ molecular emission lines with the large area observation in farinfrared (FIR) lines. We report the imaging observations of NGC 253 by FIR forbidden lines via FIS-FTS and CO molecular lines from low to high excitation levels with ASTE, which are good tracers of star forming regions or photo-dissociation regions, especially spiral galaxies, in order to derive the information of the physical conditions of the ambient interstellar radiation fields. The combination of spatially resolved FIR and sub-mm data leads to the star formation efficiency within galaxy. The ratio between the FIR luminosity and molecular gas mass, LFIR/ $\mathrm{MH}_{2}$, is expected to be proportional to the number of stars formed in the galaxy per unit molecular gas mass and time. Moreover the FIR line flux shows current star formation activity directly. Furthermore these can be systematic and statistical data for star formation history and evolution of spiral galaxies.
\end{abstract}

Key words: nearby galaxy: NGC 253; star formation; spectroscopy: FIR, sub-mm

\section{INTRODUCTION}

The objectives of this observation is to reveal the sites of star formation using suitable methods; (1) derivation of wide range spectral energy distribution (SED) from NIR to radio for various type of galaxies, (2) estimation of current starforming activity (to the star formation history in galaxies) by FIR fine structure lines, (3) sub-mm and radio data lead to physical parameters of the extended molecular gas around the disk, and consequently (4) derivation of star formation efficiency by comparison with sub-mm lines (CO) and FIR lines ([C II] ), that is, sources and results of star formation.

NGC 253 is a nearby, almost edge-on barred spiral galaxy at a distance of $2.5 \mathrm{Mpc}$, which gives a projected linear scale of $12 \mathrm{pc} / \operatorname{arcsec}$. NGC 253 is one of the nearest galaxies showing evidence for a compact nuclear starburst (size $\sim 100 \mathrm{pc}$ ) which is partially responsible for its high far-infrared luminosity, LFIR $\sim 2 \times 10^{10} \mathrm{~L} \odot$.
Then NGC 253 is a very suitable object to study the spatially resolved star formation activity in starbursts.

\section{OBSERVATIONS}

The FIR data were taken by AKARI (Murakami et al., 2007) Far-Infrared Surveyor (FIS). The AKARI AllSky Survey is used for FIR 4 band continuum. To derive line information, we used full-resolution spectroscopic mode of FIS03 (Kawada et al., 2007). To obtain more detailed spatial structure, dithering was given by multiple pointings. The line information of $\mathrm{CO}$ were derived at Nobeyama Radio Observatory (NRO) and the Atacama Submillimeter Telescope Experiment. $\mathrm{CO}(1-0)$ and $\mathrm{CO}(3-2)$ observations were carried out with the $45-\mathrm{m}$ radio telescope at NRO and the $10-\mathrm{m}$ dish at ASTE respectively. Basic On-The-Fly technique was used for both observations. Effective beam 


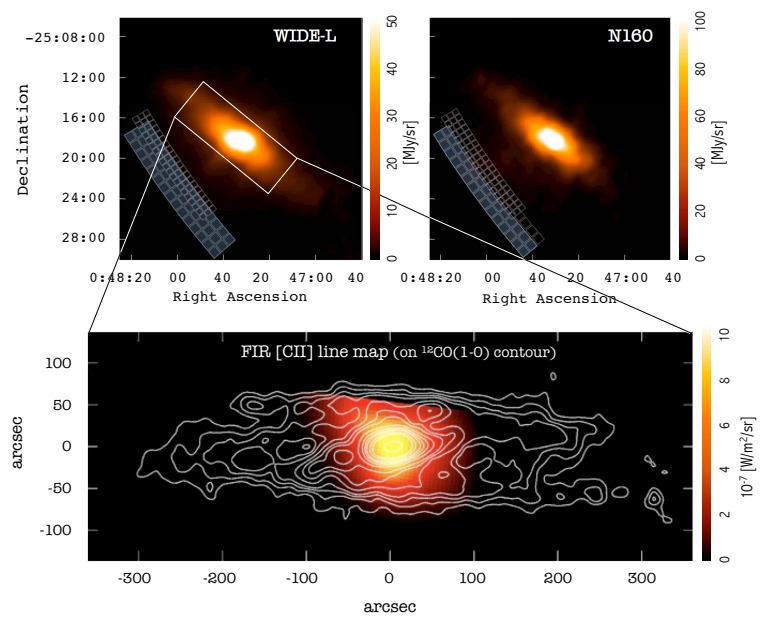

Fig. 1. FIR luminosity and [C II] flux are consistent to that of previous works, even if taking into consideration the differences of spatial coverage and resolution. The advantage of AKARI is to derive spatially resolved images.

sizes of the CO maps are $20^{\prime \prime}$ for the former and $29^{\prime \prime}$ for the latter. The beam sizes correspond to $\sim 330 \mathrm{pc}$ and $\sim 480 \mathrm{pc}$ in physical sizes at the distance of NGC 253.

\section{DISCUSSIONS}

The line ratio of $\mathrm{CO}(1-0)$ and $\mathrm{CO}(3-2)$ becomes a good index that shows the physical condition; temperature or density of molecular gas. Moreover, the star formation efficiency can be derived based on the ratio of the mass of molecular gas which is obtained by $\mathrm{CO}(1-$ 0 ) and the star formation rate from radio continuum which is synchrotron emission by supernovae and supernova remnants. In short, it is possible to derive an index of how many massive stars can be efficiently formed from a molecular gas of the same mass. On the other hand, FIR forbidden lines are emitted from ionized gas and FIR continuum represents dust temperature and total luminosity. That is, the FIR indicates the results of star formation, and on the other hand submillimeter and radio are probes of star formation. Consequently the age and evolutionary step of star formation in the center or disk of galaxies can be diagnosed from these data.

Figure 2 shows the distribution of star formation efficiency (SFE) via CO lines. It means that SFE is different within NGC 253. From Figs, [C II]/FIR is slightly low compared to active galaxies samples. Since

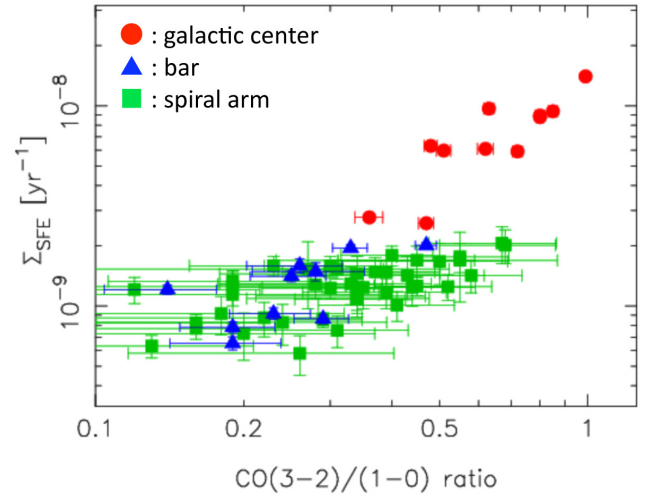

Fig. 2. The correlation between $\mathrm{CO}(3-2) / \mathrm{CO}(1-0)$ ratio and the star formation rate within galaxy appears different (Nakanishi, private communication).

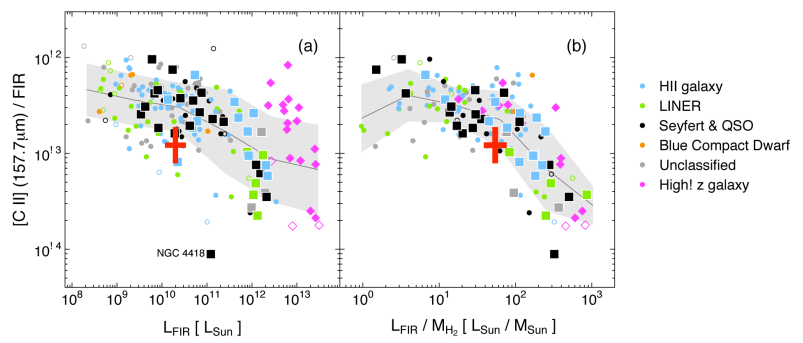

Fig. 3. Global [C II] $158 \mu \mathrm{m}$ line to FIR continuum ratio in galaxies with different optical activity classifications as a function of LFIR/MH2 (right figure). The sample of 44 galaxies are selected from Herschel/SHINING program (Gracia-Carpio et al., 2011). Cross shows NGC 253 derived this work.

there may be difference of spatial distribution of FIR continuum and $[\mathrm{C} \mathrm{II}]$ emission, it may be lack of [C II] emission from the region outside of the FOV of FIS.

\section{ACKNOWLEDGEMENTS}

This research is based on observations with AKARI, a JAXA project with the participation of ESA.

\section{REFERENCES}

Gracia-Carpio, J., et al., 2011, Far-Infrared Line Deficits in Galaxies with Extreme L FIR/MH2 Ratio, ApJ, 728, L7

Kawada, M., et al., 2007, The Far-Infrared Surveyor (FIS) for AKARI, PASJ, 59, S389

Murakami, H., et al., 2007, The Infrared Astronomical Mission AKARI, PASJ, 59, S369 